\title{
Fragile $X$ protein family member FXR1P is regulated by microRNAs
}

\author{
ANNE CHEEVER, ${ }^{1}$ ERNEST BLACKWELL, ${ }^{1,2}$ and STEPHANIE CEMAN ${ }^{1,2,3}$ \\ ${ }^{1}$ Department of Cell and Developmental Biology, University of Illinois, Urbana, Illinois 61801, USA \\ ${ }^{2}$ Program in Neuroscience, University of Illinois, Urbana, Illinois 61801, USA \\ ${ }^{3}$ College of Medicine, University of Illinois, Urbana, Illinois 61801, USA
}

\begin{abstract}
FXR1P is one of two autosomal paralogs of the fragile $X$ mental retardation protein FMRP. The absence of FMRP causes fragile $X$ syndrome, the leading cause of hereditary mental retardation. FXR1P plays an important role in normal muscle development and has been implicated in facioscapulohumeral muscular dystrophy (FSHD). Its absence also causes cardiac abnormalities in both mice and zebrafish. To examine miRNA-mediated regulation of FMRP and FXR1P, we studied their expression in a conditional Dicer knockdown cell line, DT40. We found that FXR1P, but not FMRP, is significantly increased upon Dicer knockdown and the consequent reduction of miRNAs, suggesting that FXR1P is regulated by miRNAs while FMRP is not in DT40 cells. Expression of a luciferase reporter bearing the $3^{\prime}$ untranslated region (3'UTR) of FXR1 was significantly increased in the absence of miRNAs, confirming miRNA-mediated regulation of FXR1P, while a luciferase reporter bearing the FMR1 3'UTR was not. We identified one of the regulatory regions in the 3'UTR of FXR1 by removing a conserved, 8-nucleotide miRNA seed sequence common to miRNAs 25, 32, 92, 363, and 367 and demonstrated loss of miRNA-mediated suppression. Treatment with specific miRNA hairpin inhibitors to each of the miRNAs in the seed sequence showed that miRs $92 \mathrm{~b}, 363$, and 367 regulated FXR1P expression. Accordingly, overexpression of the miRNA 367 mimic significantly decreased endogenous FXR1P expression in human cell lines HEK-293T and HeLa. We report for the first time that FXR1P is regulated through miRNA binding, with one site being the miR-25/32/92/363/367 seed sequence.
\end{abstract}

Keywords: FXR1P; miRNA; translation regulation; FMRP

\section{INTRODUCTION}

FXR1P is one member of a small family of three RNA binding proteins that also includes the fragile $\mathrm{X}$ mental retardation protein (FMRP) and FXR2P (Siomi et al. 1995; Zhang et al. 1995). Although FXR1P is found throughout the body, it is highly expressed in muscle and heart tissue where FMRP and FXR2P are mostly absent (Devys et al. 1993; Coy et al. 1995; Khandjian et al. 1995). FXR1P has seven isoforms (Kirkpatrick et al. 2001; Mientjes et al. 2004), including one cardiac-specific isoform (Khandjian et al. 1998) and three muscle-specific isoforms (Mientjes et al. 2004). FXR1P expression is essential for postnatal viability; inactivation of FXR1P in mice leads to impaired myogenesis resulting in death of neonates shortly after birth, most likely due to

Reprint requests to: Stephanie Ceman, Department of Cell and Developmental Biology, University of Illinois, 601 S. Goodwin Avenue, Urbana, IL 61801, USA; e-mail: sceman@life.illinois.edu; fax: (217) 244-1648.

Article published online ahead of print. Article and publication date are at http://www.rnajournal.org/cgi/doi/10.1261/rna.2022210. cardiac or respiratory failure (Mientjes et al. 2004). Histochemical analyses of both skeletal and cardiac muscles showed a disruption of cellular architecture and overall structure in the FXR1 knockout mice compared with wildtype (WT) littermates (Mientjes et al. 2004). In Xenopus, reduction of FXR1P disrupted MyoD expression and somite formation, while reintroduction of long and short FXR1 mRNA variants rescued these muscle-specific effects (Huot et al. 2005). In zebrafish, knockdown of FXR1 with antisense RNAs caused abnormalities in striated muscle and severe cardiomyopathy resulting in heart failure in embryos (Padje et al. 2009). Finally, in humans, altered expression of musclespecific isoforms of FXR1P has been implicated in facioscapulohumeral muscular dystrophy (FSHD) because patients have abnormal expression patterns of three different FXR1P isoforms in myoblasts and myotubes (Davidovic et al. 2008). Collectively, these studies underscore the important role of FXR1P in normal muscle development.

MicroRNAs (miRNAs) are a class of small genomically encoded RNAs that are $\sim 22$ nucleotides (nt) in size and regulate translation by base pairing with sequences in the 
$3^{\prime}$ untranslated region ( $3^{\prime} \mathrm{UTR}$ ) of target mRNA sequences (Bartel 2004, 2009). If base pairing is perfect along the $\sim 22$-nt length, the result is mRNA target degradation (Bartel 2004, 2009; Yekta et al. 2004). In contrast, if base pairing occurs in the "seed" region at the 5 ' end of the miRNA but is imperfect to the RNA, leading to a bulge in the duplex, the result is translational silencing (Bartel 2004, 2009; Jackson et al. 2009). miRNAs are estimated to regulate the expression of greater than one-third of all expressed genes (Lewis et al. 2005; Nilsen 2007); however, only a fraction of miRNAs have experimentally validated mRNA targets.

A number of miRNA target prediction programs are available to identify possible miRNA regulatory sites (Doran and Strauss 2007). Once a miRNA has been bioinformatically identified as a potential gene regulator, in vitro experiments using a luciferase reporter bearing the target sequence are commonly used to determine whether the miRNA binds the $3^{\prime}$ UTR of the target mRNA (Hurteau et al. 2007; Scott et al. 2007). To address miRNA regulation of endogenous protein expression, candidate miRNAs in the form of synthetic mimics or precursor microRNAs (pre-miRNAs) can be overexpressed to examine their effect on mRNA or protein levels (Hurteau et al. 2007; Scott et al. 2007). These approaches have been used to identify the mRNA targets of miRNAs such as $\mathrm{miR}-1$, miR-133, and miR-206, which are highly expressed in cardiac and skeletal muscle and have led to insights into normal vertebrate cardiac and skeletal muscle development and function (Chen et al. 2006, 2008, 2009).

Members of the fragile $\mathrm{X}$ family of proteins bind target mRNAs to regulate their translation (Terracciano et al. 2005); however, the molecular mechanisms underlying how FMRP or FXR1P regulate translation of their mRNA cargoes remains unclear. Both FMRP and FXR1P associate with Dicer, miRNAs, Argonaute 2 (Ago2), and other miRNA pathway components (Bartel 2004; Jin et al. 2004), suggesting that they utilize the miRNA pathway to regulate target mRNA expression. Phosphorylation of FMRP abolishes association with Dicer, the protein required for mature miRNA production, suggesting that phosphorylation regulates interaction of FMRP with the miRNA pathway (Cheever and Ceman 2009). FXR1P was implicated in translation regulation when it was shown to be recruited with Ago2 by miRNAs bound to the 3'UTR of TNF $\alpha$ mRNA in quiescent cells. The result is up-regulation of translation, suggesting that FXR1P plays a role in translation activation (Vasudevan and Steitz 2007; Vasudevan et al. 2007).

Although there is much interest in determining the mechanism by which the fragile X family of RNA binding proteins regulates expression of target mRNAs, the regulation of these translation regulators themselves is not well understood. We examined the expression of fragile $\mathrm{X}$ family members in a conditional Dicer knockdown cell line and found that FXR1P expression but not FMRP expression is regulated by miRNAs. Further, we identified an 8 -nt seed sequence in the $3^{\prime} \mathrm{UTR}$ of $F X R 1$ that is predicted to bind five different miRNAs. Expression of miRNA hairpin inhibitors against miR-92b, 363, and 367 resulted in increased FXR1P expression. In addition, overexpression of miR-367 suppressed expression of FXR1P in human cell lines HEK-293T and HeLa. Thus, we provide the first evidence that FXR1P is regulated by miRNAs.

\section{RESULTS AND DISCUSSION}

\section{Loss of Dicer expression leads to an increase in overall protein translation}

To examine the role of miRNAs in the regulation of the fragile X family of proteins, we obtained a conditional lossof-function Dicer cell line created in chicken B cell lymphoma DT40 cells (Fukagawa et al. 2004). The Dicer protein, along with associated proteins, is required for processing of pre-miRNAs into mature, functional miRNAs (Hutvagner et al. 2001). Conditional knockdown of Dicer was achieved by addition of tetracycline to the DT40 growth media every $24 \mathrm{~h}$ for $96 \mathrm{~h}$ as previously described (Fukagawa et al. 2004), at which time a significant loss of Dicer expression was observed (Fig. 1A, top panel). To understand the effects of Dicer and subsequent miRNA knockdown on protein translation in the DT40 cells, we examined polyribosome (polysome) profiles in the presence (-tet) and absence (+tet) of

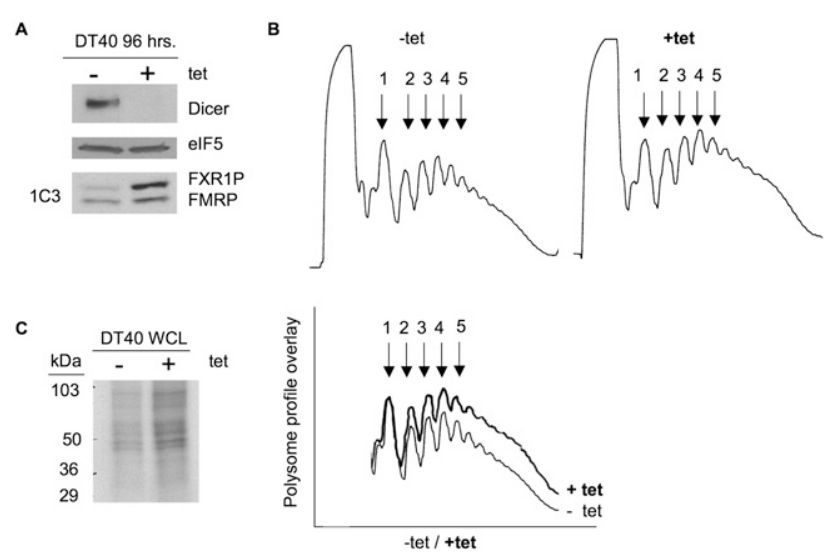

FIGURE 1. Loss of Dicer expression leads to a global increase in translation. $(A)$ Protein extracts from DT40 cells grown in the absence $(-)$ or presence $(+)$ of tetracycline (tet) for $96 \mathrm{~h}$ were examined by immunoblot for the proteins indicated on the right. (Bottom panel) Antibody 1C3 was used to detect FXR1P and FMRP. (B) Polysomes from DT40 cells were harvested after $96 \mathrm{~h}$ of growth in the absence (-tet) or presence (+tet) of tetracycline and analyzed by sucrose gradient fractionation and measured at absorption of $254 \mathrm{~nm}$. (1) mRNAs with one ribosome, (2) mRNAs with two ribosomes, etc. (Lower left) Overlay of the profiles. Percentage increase in the area under the curve of polysomes grown in the presence of tetracycline compared with its absence (42\%) was determined using Image J. (C) $\left[{ }^{3} \mathrm{H}\right]$-methyl-methionine, pulse-labeled protein extracts from DT40 cells grown for $96 \mathrm{~h}$ in the absence $(-)$ or presence $(+)$ of tetracycline were resolved on a 4\%-20\% SDS-PAGE gel. Molecular weight markers are shown at the left $(\mathrm{kDa})$. 
Dicer (Fig. 1B). Polysomes are mRNAs with two, three, four, etc., ribosomes associated with them, reflecting the translational state of the cell, and are visualized by UV absorption of fractionated lysates at $254 \mathrm{~nm}$ (Ruan et al. 1997). Examples of polysome profiles from mock-treated DT40 cells and cells treated with tetracycline are shown in Figure 1B. In the presence of Dicer (-tet), none of the polysome peaks were as high as the monosome peak (peak 1), which is typical (Khandjian et al. 1996; Feng et al. 1997; Ruan et al. 1997). In contrast, in the absence of Dicer (+tet) and, therefore, mature miRNA production, we reproducibly observed an increase in the height of the polysome peaks, suggesting that there was an increase in translation initiation in the absence of miRNAs (Fig. 1B, bottom, cf. +tet and -tet). The percent increase in total area of the polysome profiles in the presence of tetracycline (+tet) was $42 \%$ compared with its absence (Fig. 1B, bottom). To verify that there was more protein synthesis in the absence of Dicer and miRNAs, we measured new protein synthesis in similarly treated DT40 cells by $\left[{ }^{3} \mathrm{H}\right]$-methionine incorporation and found an increase in the amount of labeled proteins $(40 \%$ as measured by TCA incorporation) (Fig. $1 \mathrm{C}$; data not shown), further indicating increased protein synthesis in the absence of miRNAs.

\section{FXR1P but not FMRP is significantly increased in the absence of Dicer in DT40 cells}

Proteins whose syntheses are increased in the absence of Dicer and miRNAs are potential candidates for miRNA-mediated regulation. To explore the regulation of FXR1P and FMRP, we probed cell extracts for FMRP and FXR1P using monoclonal antibody 1C3 (Devys et al. 1993), which recognizes both FMRP and FXR1P in mammalian cells (Mazroui et al. 2003). By immunoblot, we observed a significant increase in the abundance of the top band, but very little change in the bottom band (Fig. 1A, bottom panel), suggesting an overall increase in translation of either FXR1P or FMRP. Since DT40 is a chicken B cell line, we suspected that FXR1P was the top band and that FMRP was the bottom band because both chicken and zebra finch FMRP are missing exons 11 and 12 (Price et al. 1996; Winograd et al. 2008); we therefore predicted that FMRP migrated faster in the gel and was the smaller-sized protein. To verify the iden- tity of the proteins visualized by antibody 1C3 (Devys et al. 1993) in the DT40 cell line, we immunoprecipitated DT40 cellular extracts with an antibody specific to FXR1P and showed by 1C3 immunoblot that FXR1P was the top band (Fig. 2A). Similarly, immunoprecipitation with two FMRPspecific antibodies (Abcam) followed by 1C3 immunoblot showed that FMRP was the bottom band (Fig. 2B).

We consistently found an increase in FXR1P expression in the absence of Dicer and miRNAs. On average, FXR1P showed a fourfold increase in the absence of Dicer (Fig. 2C,D). In contrast, although there was a trend toward increased FMRP expression, it was not statistically significant (Fig. 2D). To investigate whether increased protein expression of FXR1P was mediated by enhanced translation in the absence of Dicer, we pulse-labeled cells with $\left[{ }^{3} \mathrm{H}\right]$-methionine to visualize newly synthesized FXR1P and FMRP. In the absence of miRNAs,
A

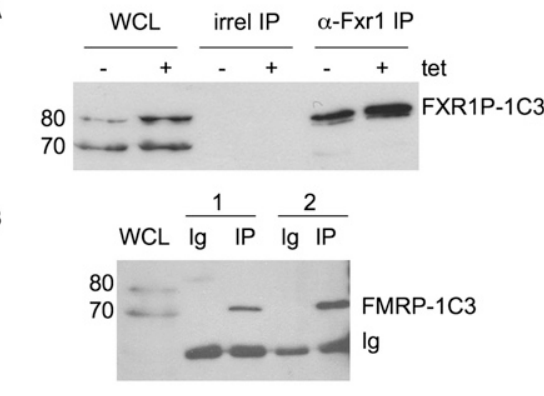

C

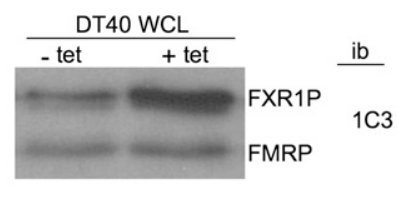

D

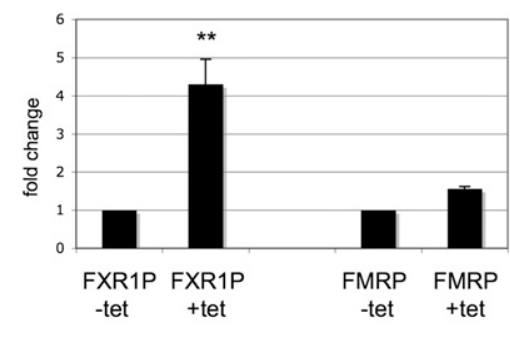

E

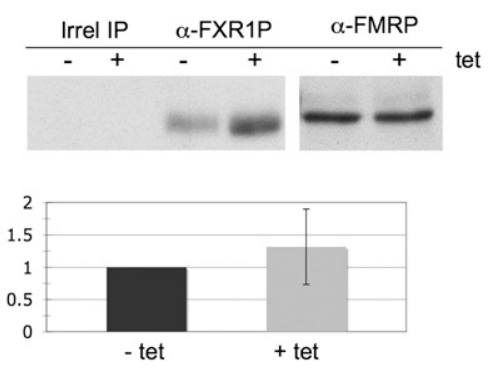

FIGURE 2. FXR1P but not FMRP levels significantly increase in the absence of Dicer, while FXR1 mRNA levels do not significantly change. (A) DT40 cells were grown for $96 \mathrm{~h}$ in the absence $(-)$ or presence $(+)$ of tetracycline, immunoprecipitated with an irrelevant antibody or an anti-FXR1P-specific antibody (Jin et al. 2004) as indicated, and immunoblotted with antibody 1C3 (Devys et al. 1993). (WCL) Whole cell lysate. (B) Immunoprecipitation with two independent FMRP-specific antibodies (Abcam) and subsequent immunoblot with antibody 1C3 (Devys et al. 1993). (Ig, immunoglobulin) Immunoprecipitating antibody alone (above blot) or reactivity of HRP detecting antibody to Ig chains (at right). (C) Representative immunoblot of DT40 cells harvested after $96 \mathrm{~h}$ in the absence or presence of tetracycline (-tet, tet, respectively) and probed with 1C3 antibody (Devys et al. 1993). Positions of FXR1P and FMRP are indicated (right). (D) Quantification of fold change of FXR1P and FMRP in the presence and absence of tetracycline as indicated. Significance was determined using Students's $t$-test. $\left.{ }^{* *}\right) P$-value $<0.01, n=3$. No statistically significant change in FMRP expression after addition of tetracycline was found ( $P$-value $=0.267$ using Student's $t$-test, $n=3$ ). $(E)$ Autoradiographs of FXR1P or FMRP immunoprecipitations from $\left[{ }^{3} \mathrm{H}\right]$-methyl-methionine pulse-labeled DT40 cells after $96 \mathrm{~h}$ of $-/+$ tetracycline. Immunoprecipitating antibodies are indicated (above). (F) Quantitative real-time PCR using triplicate $\Delta \mathrm{Ct}$ values from three independent trials of RNA extracted and reverse transcribed from DT40 cells grown for $96 \mathrm{~h}$ in the presence or absence of tetracycline (+tet and -tet, respectively) was used to calculate the $2^{-\Delta \mathrm{Ct}}$ and normalized to FMR1 mRNA levels. Values of FXR1 transcript in the absence of tet are indicated as 1 , and fold change in the presence of tetracycine is indicated. Lack of significance was assessed by Z-test $(P$-value $=0.307, n=3)$. 
there was more FXR1P synthesized compared with FMRP, whose translation levels remained the same (Fig. 2E). These results provide the first evidence for translational regulation of FXR1P, likely through the miRNA pathway.

\section{The absence of Dicer has no effect on FXR1 mRNA levels}

In addition to suppressing translation, miRNAs can regulate protein expression by causing mRNA degradation (Bartel 2004; Nilsen 2007; Filipowicz et al. 2008; Lal et al. 2009). To assess whether FXR1P expression is regulated at the mRNA level, FXR1 mRNA levels in the presence and absence of miRNAs were compared. We performed quantitative reversetranscription PCR on total RNA isolated from DT40 cells that were treated with tetracycline (+tet) or mock treated (-tet) for $96 \mathrm{~h}$ (Fig. 2F). The FXR1 primer set was specific to exon 5 in FXR1 mRNA, which is present in all of the seven FXR1 splice isoforms (Kirkpatrick et al. 1999; Dube et al. 2000). We measured the change in cycle threshold values $(\Delta \mathrm{Ct})$, which is the point where the increases in cDNA become exponential and cross the threshold of detection. Although there appears to be a trend toward increased FXR1 transcript levels in the absence of Dicer and miRNAs, we found no statistically significant difference in FXR1 mRNA levels in Dicer knockdown cells (Fig. 2F). Because miRNAs have been shown to regulate protein levels by inducing mRNA degradation, we might have expected to observe a change in mRNA levels; however, we did not. Thus, our results suggest that the increase in FXR1P expression in the absence of Dicer is likely due to a relief of miRNA-mediated translation suppression.

\section{The 3'UTR of FXR1 contains putative miRNA regulatory sites}

To identify potential miRNA regulatory sites in the $3^{\prime}$ UTR of FXR1, we used TargetScan 5.1 to predict miRNA binding sites (Fig. 3A). Each box represents the seed sequence of a single miRNA or the seed sequence shared by a group of miRNAs, as indicated (Fig. 3A). Two of the predicted sites, miR-148 and miR-25/32/92/363/367, each contained an 8-nt conserved sequence and had the highest probability of preferential conservation (http://www.targetscan.org). In addition, we considered the position of the putative miRNA binding site in the context of the entire FXR1 3'UTR (Grimson et al. 2007; Majoros and Ohler 2007). Since functional miRNA regulatory sites are preferentially found away from the center of $3^{\prime}$ UTRs, at least $15 \mathrm{nt} 3^{\prime}$ from the stop codon and/or near the polyadenylation sequence (Grimson et al. 2007; Majoros and Ohler 2007; Gu et al. 2009), we chose to focus on the highly conserved, shared seed sequence of miRNAs $25,32,92$, 363 , and 367. Finally, all FXR1 isoforms maintain the same 3'UTR (Kirkpatrick et al. 1999; Dube et al. 2000). Thus, all of the FXR1P spliceforms could potentially be regulated by miRNA binding at the $3^{\prime}$ UTR.

\section{Candidate miRNAs miR-92 and miR-363 are expressed in DT40 cells}

We used Northern blotting to examine whether any of these five candidate miRNAs-25, 32, 92, 363, and 367-were expressed in DT40 cells (Fig. 3B,C). miR-92 and miR-363 were expressed at detectable levels in DT40 cells and were present as pre-miRNAs in the absence of Dicer (Fig. 3B,C, + lanes), as expected. Accordingly, in the presence of Dicer $(-$ tet), these pre-miRNAs were processed into mature miRNAs (Fig. 3B,C). In contrast, we were unable to detect candidate miRNAs 32 or 367 (data not shown), indicating that they were either not expressed in DT40 cells or were below the detection limit of Northern blotting. miR-25 was not tested. Additionally, miRNAs 30a, 30a-5p, 31, 301, 199b, 200b, 429, and 124a (used as a negative control) were not detected in DT40 cells (data not shown). Since miRNAs corresponding to the conserved miR-25/32/92/363/367 site were expressed and regulated by the presence or absence of Dicer, we concluded that this site could potentially regulate FXR1P expression.

\section{The 3'UTR of FXR1 confers miRNA-mediated regulation on a luciferase reporter construct}

To test our hypothesis that miRNAs regulate expression of FXR1P through its 3'UTR, we expressed the 3'UTR from either FXR1 or FMR1 downstream from the luciferase open reading frame in the pGL3 expression vector. We transfected these constructs into DT40 cells treated with tetracycline $(+)$ or mock treated (-) and quantified luciferase expression. Interestingly, introduction of the $3^{\prime}$ UTR from either FXR1 or FMR1 increased luciferase expression compared with the empty vector alone (pGL3) (Fig. 3D,E). Importantly, we found that in the absence of Dicer (-) and consequently miRNAs, luciferase expression driven by the FXR1 3'UTR was significantly increased compared with its expression in the presence of Dicer (+) (Fig. 3D, cf. +Dicer FXR1 and - Dicer FXR1). These results suggest that the 3'UTR of FXR1 is regulated by miRNAs. The relative increase in luciferase expression in the absence of Dicer in Figure 3D is not as high as the relative increase in endogenous FXR1P expression under the same conditions, as shown in Figure 2D. One possible explanation is that there are additional miRNA regulatory sites within either the coding region of FXR1P, as has been described for other genes (Rigoutsos 2009), or in the $5^{\prime}$ UTR, as has been generated in vitro (Lytle et al. 2007). In addition, the approach used to clone the $3^{\prime} \mathrm{UTR}$ of FXR1 excluded $46 \mathrm{nt}$ from the end of the $3^{\prime} \mathrm{UTR}-\mathrm{a}$ region predicted to contain a putative miR-7 site (Fig. 3A), which has a lower probability of preferential conservation compared with some of the other sites (TargetScan5.1). This site would be present in the endogenous gene but not in the luciferase construct and may contribute to translation suppression in DT40 cells in the absence of tetracycline and presence of 


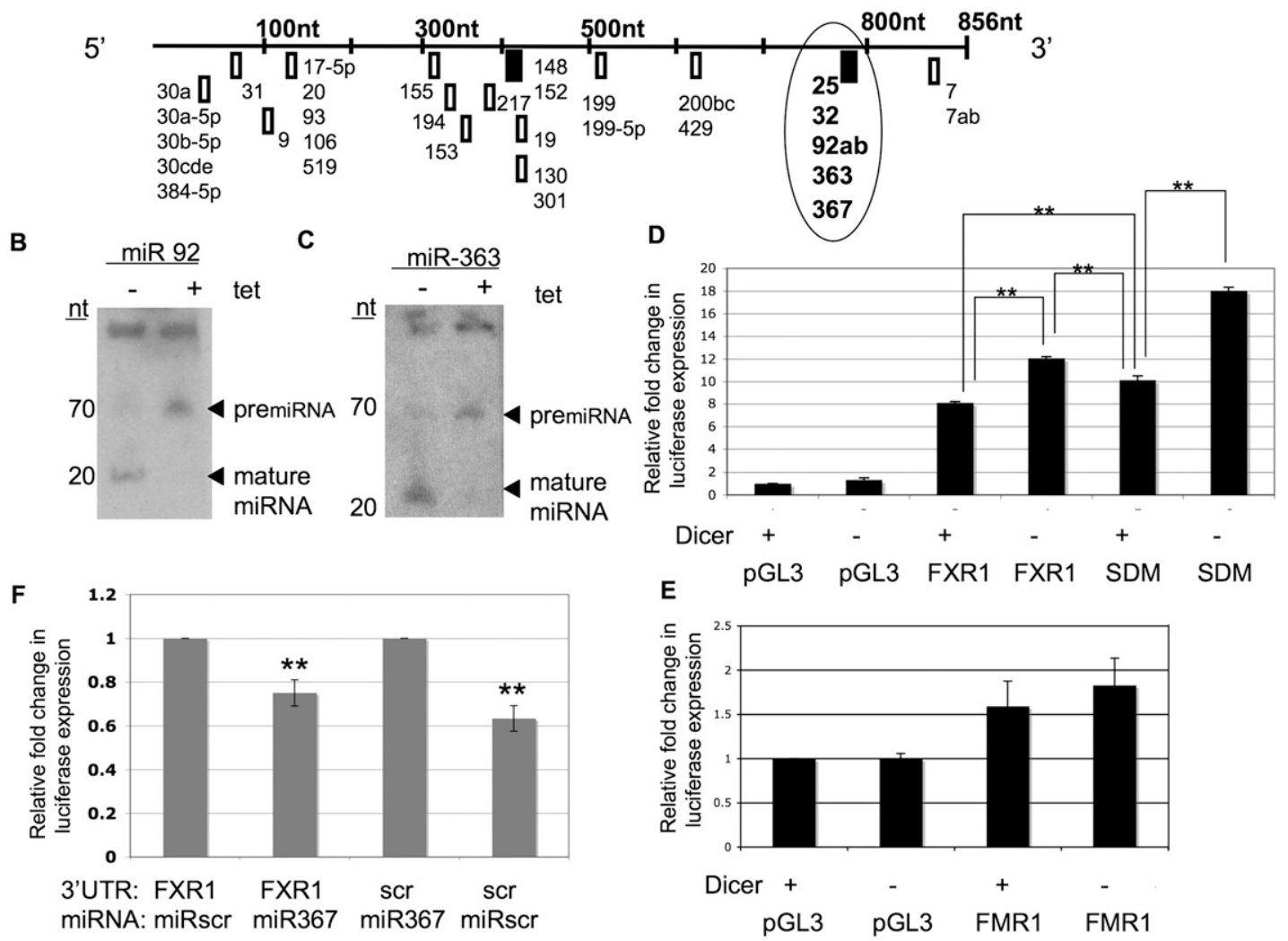

FIGURE 3. FXR1 3'UTR contains a common seed sequence that confers miRNA-mediated regulation on luciferase constructs. (A) Schematic of the predicted miRNA seed sequence binding sites in the FXR1 3'UTR based on TargetScan 5.1. miRNAs are labeled. (Boxes) Seed sequences, (bold boxes) the two 8-nt sites with higher probability of preferred conservation, (circle) location of the 8-nt seed sequence common to miRNAs-25/32/ 92/363/367. miR-7 was predicted by TargetScan 5.1 but was not included in the FXR1 3'UTR subcloned luciferase constructs. (B,C) Total RNA $(50 \mu \mathrm{g})$ from DT40 cells treated with tetracycline for $96 \mathrm{~h}(+)$ or mock treated $(-)$ was resolved on a $15 \%$ acrylamide gel and probed with $\left[{ }^{32} \mathrm{P}\right]-$ labeled antisense oligos to miR-92 (B) and miR-363 (C), as indicated (top). (Left) Size in nucleotides, (right) positions of pre-miRNAs (upper band) and mature miRNAs (lower band). (D) pGL3 luciferase reporter vector alone (pGL3), containing the 3'UTR of FXR1 (FXR1), or containing the FXR1 3'UTR in which the seed sequence common to miRNAs 25/32/92/363/367 was deleted (SDM) were electroporated into DT40 cells after $72 \mathrm{~h}$ growth in tetracycline. Twenty-four hours after electroporation (for 96 total hours in tetracycline), luciferase expression was assessed in DT40 cells grown in the presence $(+)$ or absence $(-)$ of tetracycline (tet). Transfection efficiency was monitored by Renilla expression, and all luciferase values were normalized to empty vector (pGL3) in the absence of tetracycline. Values are depicted as expression fold change compared with empty vector. Significance was determined by Student's $t$-test. $\left.{ }^{* *}\right) P$-value $<0.007, n=3$. (E) pGL3 vector containing the FMR1 $3^{\prime}$ UTR was electroporated into DT40 cells under the conditions described above. No significant difference in luciferase expression was observed in the presence $(-)$ or absence $(+)$ of miRNAs by Student's $t$-test $(P$-value $=0.392, n=3) .(F)$ pGL3 vector containing the FXR1 3'UTR or the FXR1 3'UTR in which the 25/32/92/363/367 was deleted and replaced with the sequence CCTGTTAG (scr) were introduced into HEK293 cells with either the miR367 mimic or the miRNA complementary to the CCTGTTAG (scr) sequence (miR-scr), as indicated below. Luciferase and Renilla activity was measured and ratios calculated. Values are depicted as expression fold change determined by comparing luciferase expression in the presence of the complementary miRNA to luciferase expression in the presence of the opposite, nonbinding miRNA, the latter value designated as 1. Relative fold change is indicated. Significance was determined by Student's $t$-test. $\left.{ }^{* *}\right) P$-value $<0.01, n=3$.

miRNAs. Finally, the location of the 25/32/92/363/367 seed sequence relative to the end of the $3^{\prime} \mathrm{UTR}$ and the polyadenylation site differs between the endogenous FXR1 mRNA and the luciferase construct, which contains an SV40 polyadenylation site. This disparity may affect the efficacy of the seed sequence, as proximity to the polyadenylation site has been determined to play a role in target site usage by miRNAs (Grimson et al. 2007; Majoros and Ohler 2007; Gu et al. 2009).

In contrast to the miRNA-dependent regulation of the 3'UTR of FXR1, there was no statistically significant increase in expression of the FMR1 luciferase construct in the absence of miRNAs (Fig. 3E, cf. +Dicer FMR1 and -Dicer FMR1). These results suggest that in DT40 cells the 3'UTR of FXR1 is regulated by miRNAs and the $3^{\prime} \mathrm{UTR}$ of FMR1 is not. Unlike FMR1, FXR1 orthologs have highly conserved 3'UTRs $(>90 \%)$, suggesting an important regulatory function (Siomi et al. 1995). Further evidence that FXR1, and not FMR1, is regulated by miRNAs comes from a recent study showing that the FXR1 mRNA, but not the FMR1 mRNA, is found associated with Ago2 in HEK-293T cells, suggesting that the FXR1 mRNA is regulated by miRNAs (Hendrickson et al. 2008). Similarly, high-throughput sequencing of mRNAs 
crosslinked to Ago2 failed to detect the FMR1 mRNA (FXR1 was not tested) (Chi et al. 2009). Even though many miRNA binding sites are predicted in the FMR1 3'UTR, none of the miRNAs tested bound the $3^{\prime} \mathrm{UTR}$, indicating a lack of functional miRNA binding (Chi et al. 2009). Taken together, these findings support our data showing that expression of FXR1P, but not FMRP, is regulated by miRNAs in the DT40 cells.

To determine if the miRNA-mediated suppression of luciferase expression occurs through the 8-nt seed sequence circled in Figure 3A, we deleted this sequence and examined the effect on luciferase expression. Deletion of this site significantly increased luciferase expression in the presence of miRNAs compared with the WT 3'UTR (Fig. 3D, cf. +Dicer SDM and +Dicer FXR1), suggesting that miRNAs bind that site in the full-length construct and suppress it. However, removal of the site did not relieve suppression as much as reducing miRNA expression did (Fig. 3D, cf. +Dicer SDM and -Dicer FXR1). Thus, removal of the miRNA binding site significantly relieved some, but not all, of the miRNAmediated suppression. This result suggests that there are additional sites in the FXR1 $3^{\prime}$ UTR that are regulated by miRNAs. Finally, simultaneous removal of the 8-nt seed sequence and reduction of miRNAs by loss of Dicer resulted in the most luciferase expression (-Dicer SDM). We suspect that this construct is the most highly expressed because residual miRNAs may still be present (even though Dicer is absent) that are still able to bind the 25/32/92/363/367 site. When the 8-nt seed sequence is removed, the residual miRNAs can no longer bind, leading to the most derepressed condition and the most luciferase expression.

To demonstrate a direct effect of miRNA binding through the 25/32/92/363/367 seed sequence site we introduced a novel sequence (scr) at that site that is not bound by any miRNAs, confirmed using miRBase (http://www.mirbase.org). We then co-transfected the FXR1 luciferase reporter with either the miR367 mimic, which is predicted to bind the 25/32/92/363/ 367 seed sequence (Fig. 3A), or the miRNA directed to the novel sequence (miR-scr). As expected, miR-367 suppressed luciferase expression $25 \%$ from the FXR 1 construct compared with coexpression of miR-scr, which was normalized to 1 (Fig. $3 \mathrm{~F}$, left). Accordingly, co-transfection of the scr-substituted luciferase reporter (scr) with miR-scr suppressed luciferase expression $37 \%$ compared with the scr-luciferase construct expressed with miR-367, which was normalized to 1 (Fig. 3F, right). We conclude that translation is regulated via base pairing of miRNAs to the 25/32/92/363/367 site. In summary, we have demonstrated that the $3^{\prime} U T R$ of $F X R 1$ is regulated by miRNA binding to the $25 / 32 / 92 / 363 / 367$ site.

\section{FXR1P expression is regulated by miR-92b, miR-363, and $\mathrm{miR}-367$}

To identify the miRNAs that regulate FXR1P expression in human cells, we introduced hairpin inhibitors to each of the miRNAs in the $25 / 32 / 92 / 363 / 367$ seed sequence (Fig. $3 \mathrm{~A})$. All of the miRNAs predicted to bind the seed sequence are present in HEK-293T cells (Landgraf et al. 2007). We found that inhibitors directed to miR-92b, miR-363, and miR-367 significantly increased FXR1P expression (Fig. 4A,B), suggesting that miR-92b, miR-363, and miR-367 regulate FXR1P expression. In contrast, inhibitors to miRs 25, 32, and 92a had no significant effect on FXR1P expression. In addition, treatment with a negative control RNA hairpin, Cel-miR-67, had no significant effect on FXR1P expression (Fig. 4C,D). Thus, although all six miRNAs in the conserved seed sequence are expressed in HEK-293T cells, only miR92b, miR-363, and miR-367 regulate FXR1P expression.

Because miRNA inhibition resulted in enhanced FXR1P expression, we hypothesized that overexpression of any of the seed sequence miRNAs would suppress FXR1P expression. Accordingly, HeLa and HEK-293T cells were transfected with a double-stranded miR-367 miRNA mimic and harvested after 72 or $96 \mathrm{~h}$ (Fig. 4E,F). After assessing FXR1P expression, quantification showed a $\sim 60 \%$ reduction in FXR1P expression levels compared with control in HeLa cells (Fig. 4E). In HEK-293T cells, there was an average reduction in FXR1P expression $>60 \%$ compared with the control (Fig. 4F). Transfection of an irrelevant siRNA did not affect FXR1P expression in either cell line (data not shown). Thus, expression of a miRNA specific to the seed sequence miR-25/32/92/363/367, namely miR367, is sufficient to significantly down-regulate expression of multiple FXR1P splice forms in two human cell lines.

Regulation of FXR1P expression by miRNAs is an important finding because although miRNAs have been shown to regulate key transcription factors such as MYC, E2Fs, and MYB (Xiao et al. 2007; Lal et al. 2009), there are very few reports of miRNA-mediated regulation of RNA binding proteins. One example is HuR, a sequence-specific RNA binding protein that regulates translation and RNA turnover, and in turn influences the cellular response to stress, proliferative signals, immune triggers, and developmental cues (Gorospe 2003; Lopez de Silanes et al. 2005; Cherry et al. 2006; Abdelmohsen et al. 2007). HuR was recently shown to be regulated by miR-519 (Abdelmohsen et al. 2008). We now add FXR1P as an additional example of one posttranscriptional regulatory factor (an RNA binding protein) being regulated by another type of post-transcriptional regulator (miRNAs).

FXR1P is implicated in normal muscle development and function (Mientjes et al. 2004; Huot et al. 2005), as are miRNAs (Wienholds and Plasterk 2005; Chen et al. 2006, 2009). In fact, comprehensive miRNA expression-profiling studies revealed that a total of 185 miRNAs was dysregulated in samples of diseased muscle tissue from 10 different muscle disorders (Eisenberg et al. 2009). miR-92, a candidate miRNA for FXR1P regulation, was found to be misregulated in Duchennes' Muscular Dystrophy and in Nemaline Myopathy, a congenital myopathy that is the most common 


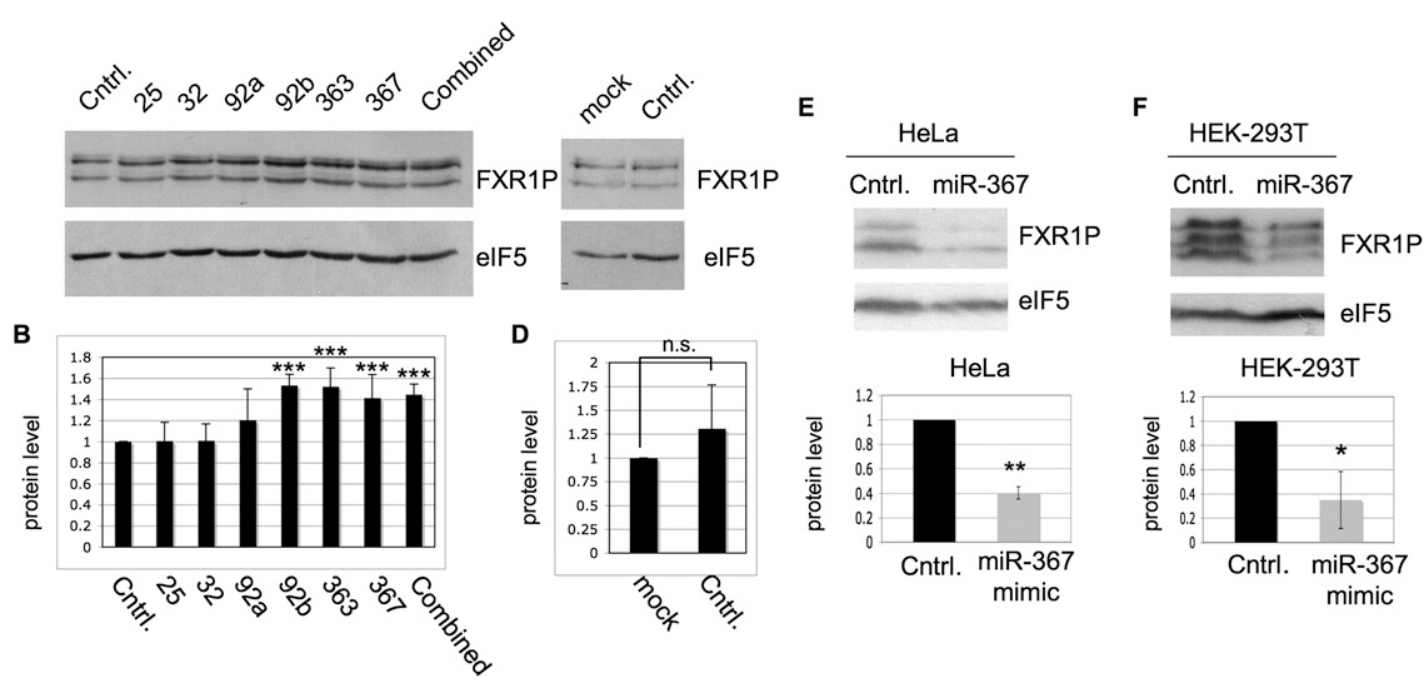

FIGURE 4. Inhibition of specific miRNAs 92b, 363, and 367 increases FXR1P expression while overexpression of miR-367 reduces FXR1P expression. (A) HEK-293T cells were transfected with either the negative control miRNA inhibitor (Cntrl.), a single miRNA inhibitor for miR-25, miR-32, miR-92a, miR-92b, miR-363, or miR-367, or all six miRNA inhibitors at equal concentrations (combined). FXR1 protein levels were assessed by immunoblot and eIF5 was used as a loading control. (B) Densitometry was performed using Image J and the Z-test was used to determine significance. $\left.{ }^{* * *}\right) P$-value $<0.001, n=5$. miRs 25,32 , and 92a were not significantly different from the control inhibitor $(P$-values $0.47,0.465$, and 0.062 , respectively, $n=5$ ). (C) HEK-293T cells were transfected with either the negative control miRNA inhibitor (Cntrl.) or mock transfected (mock) and FXR1 protein levels were assessed by immunoblot with eIF5 as a loading control. $(D)$ Densitometry was performed using Image $\mathrm{J}$ and lack of significance calculated using the Z-test $(P$-value $=0.066, n=5)$. HeLa $(E)$ and HEK-293T $(F)$ cells were transfected with the miR-367 mimic. Seventy-two or 96 h post-transfection, mimic-transfected cells (miR-367) or mock-transfected cells (Cntrl.) were harvested and probed for FXR1P and eIF5, as indicated $(r i g h t)$ in top panels $(E, F)$. Densitometry was performed using Image J. FXR1P expression was normalized to the eIF5 loading control before quantifying FXR1P expression on three independent experiments. Significance was calculated by Student's $t$-test. $\left.{ }^{*}\right) P$-value $<0.05,\left({ }^{*}\right) P$-value $<0.01$.

nondystrophic congenital myopathy (Eisenberg et al. 2009). Thus, it is formally possible that FXR1P, and consequently its target mRNAs, is misregulated in muscle disease states as a result of misregulated miRNA expression. Understanding how FXR1P is regulated by miRNAs may provide crucial insight into normal muscle development and function.

\section{MATERIALS AND METHODS}

\section{Cell lines and antibodies}

The DT40 cell line was maintained between $10^{5}$ and $10^{6}$ cells per mL in Dulbecco's modified medium (Sigma D5796) supplemented with $10 \%$ tetracycline-free fetal calf serum (Clontech), $1 \%$ chicken serum, $1 \%$ penicillin/streptomycin (Gibco), and $10 \mu \mathrm{M} \beta$-mercaptoethanol (Fisher) (Buerstedde and Takeda 1991). HeLa and HEK293 cell lines were maintained in Dulbecco's modified medium supplemented with $10 \%$ fetal calf serum, $1 \%$ penicillin/streptomycin, and $1 \%$ nonessential amino acids. Anti-FMRP antibody IAC-1C3, hereafter referred to as monoclonal antibody 1C3 (Devys et al. 1993), was used to detect both FMRP and FXR1P in immunoblots where indicated at a 1/10 dilution of the hybridoma supernatant. Anti-FXR1P 2107 was used to detect FXR1P in HeLa and HEK-293T cells by immunoblot as described (Bakker et al. 2000). Anti-FXR1 was used to immunoprecipitate FXR1P as described (Jin et al. 2004). Anti-eukaryotic initiation factor 5 (eIF5) (Santa Cruz) was used for loading controls.
Rabbit anti-Dicer (Santa Cruz) was used to detect Dicer. Antibody reactivity was visualized using either an anti-mouse HRP conjugate (Jackson Immunoresearch) or an anti-rabbit HRP conjugate (GE Healthcare) and developed with ECL (GE Healthcare).

\section{Dicer knockdown}

DT40 cells were cultured as previously described and maintained at $6 \times 10^{5}$ cells $/ \mathrm{mL}$ for $2 \mathrm{~d}$ before addition of tetracycline dissolved in $\mathrm{EtOH}$ at a final concentration of $2 \mu \mathrm{g} / \mathrm{mL}$ of media. Freshly made tetracycline (+tet) or vehicle only (EtOH, -tet) was added to cell media every $24 \mathrm{~h}$ until cells were harvested at $96 \mathrm{~h}$. Dicer knockdown was confirmed by immunoblot with Dicer antibody.

\section{Metabolic labeling}

DT40 cells grown as described above for $96 \mathrm{~h}$ were pre-incubated in methionine-free media [Gibco] supplemented with glutamine and $10 \%$ dialyzed FCS for $30 \mathrm{~min}$ before introducing $50 \mu \mathrm{Ci} / \mathrm{mL}$ $\left[{ }^{3} \mathrm{H}\right]$-methyl-methionine (Amersham) for $45 \mathrm{~min}$. The cells were then washed twice with ice-cold PBS and lysed with lysis buffer (50 $\mathrm{mM}$ Tris pH 7.6, $300 \mathrm{mM} \mathrm{NaCl}, 30 \mathrm{mM}$ EDTA, $0.5 \%$ Triton X-100, and a protease inhibitor tablet [Roche]) for $10 \mathrm{~min}$. Cytoplasmic extracts were prepared by spinning to remove the nuclei, and either total extract was loaded or the lysate was immunoprecipitated with the anti-FXR1P antibody (Jin et al. 2004) or anti-FMRP antibodies (Abcam) as described (Stetler et al. 2006). 


\section{Linear sucrose density gradient analysis of polysomes}

DT40 cells grown as described above were treated for $15 \mathrm{~min}$ with cycloheximide $(100 \mu \mathrm{g} / \mathrm{mL})$. Linear $15 \%-45 \%$ sucrose gradients containing $100 \mathrm{mM} \mathrm{KCl}, 20 \mathrm{mM}$ Tris ( $\mathrm{pH} 7.5$ ), and $5 \mathrm{mM} \mathrm{MgCl}_{2}$ were prepared using a gradient maker (BioComp). Cells were washed in PBS and lysed in the buffer described above. Postnuclear supernatants were overlayed on the gradient and centrifuged for $75 \mathrm{~min}$ at $188,000 \mathrm{~g}$ at $4^{\circ} \mathrm{C}$. Each gradient was fractionated into $1-\mathrm{mL}$ fractions by bottom displacement using a gradient fractionator (Isco) with the ribosomal profile monitored at $254 \mathrm{~nm}$. The total area under the polysome profiles was calculated using Image J.

\section{RNA isolation and quantitative reverse transcription-polymerase chain reaction}

Total RNA was isolated using TRIzol (Invitrogen) and reverse transcribed using random primers and superscript II reverse transcriptase (Invitrogen). qRT-PCR was performed and $\Delta$ Ct values were calculated and averaged using triplicate samples from each of three independent trials in a BioRad iCycler using QuantiTect SYBR green (Qiagen). PCR was performed by initial denaturation for $15 \mathrm{~min}$ at $95^{\circ} \mathrm{C}$ followed by 40 cycles of $15 \mathrm{sec}$ at $94^{\circ} \mathrm{C}, 30 \mathrm{sec}$ at $55^{\circ} \mathrm{C}$, and $30 \mathrm{sec}$ at $72^{\circ} \mathrm{C}$. The primers for FXR1 and $F M R 1$ were validated and obtained from Qiagen using the QuantiTect Primer Assay System (Cat. Nos. QT01498000 and QT01501031, respectively). Primer specificity was verified by melt curve analysis, and fold change between FMR1 mRNA levels (internal control for mRNA level normalization) and FXR1 mRNA levels was calculated as $2^{-\Delta \mathrm{Ct}}$. Lack of significance was assessed by a Z-test showing no significant difference between the FXR1 mRNA level fold change and 1.

\section{Reporter gene assay and cell electroporation}

The 3'UTRs for chicken FXR1 and FMR1 were cloned from total DT40 RNA by RT-PCR for insertion into the XbaI site located $3^{\prime}$ of the luciferase open reading frame in pGL3 (Promega). Primers: FXR1-F-gctctagacctgaatggtgtttc; FXR1R-gctctagacatattgcacatcaaac aacc; FMR1-F-gctctagaataagtctgaagtcat; FMR1-R-gcttctagattgaggct aacctactg. At $72 \mathrm{~h}, 10^{7}$ DT40 cells, treated as described above, were transfected by electroporation with $25 \mu \mathrm{g}$ of reporter constructs (pGL3-derived) containing the firefly luciferase gene and either the chicken FXR1 3'UTR, the chicken FMR1 3'UTR, or the mutated chicken FXR1 3'UTR from which the seed sequence was removed using site-directed mutagenesis (Stratagene) and $10 \mu \mathrm{g}$ of the Renilla luciferase phRL-Tk vector (Promega). The substituted construct (scr) was made by introducing CCTGTTAG into the 25/32/92/363/367 site. Following electroporation, DT40 cells were immediately transferred to individual T75 cell culture flasks, treated with either tetracycline or vehicle only $(\mathrm{EtOH})$, and grown for an additional $24 \mathrm{~h}$ at which time $(96 \mathrm{~h}$ after initial tetracycline/EtOH treatment) $3 \times 10^{6}$ cells were assayed for luciferase expression and the remaining cells were harvested for Dicer expression by immunoblot. The study shown in Figure 3F was carried out by transfecting HEK-293T cells with the constructs indicated and a miRNA mimic to the site-substituted sequence (scr) (Dharmacon). Luciferase assays were carried out using the Dual-Luciferase Reporter Assay (Promega) according to the manufacturer's directions. Luciferase and Renilla activity were measured in a LUMIstar OPTIMA Luminometer programmed with OPTIMA software version 2.00.

\section{Inhibition of miRNAs using miRNA inhibitors}

miRIDIAN miRNA hairpin inhibitors (Dharmacon) were used to inhibit miRNA expression following the manufacturer's instructions. Briefly, HEK-293T cells were plated at $2.5 \times 10^{4}$ in 24 -well plates. Twenty-four hours after plating, cells were transfected with miRNA inhibitors at a final concentration of $100 \mathrm{nM}$ using DharmaFECT 1 transfection reagent. Twenty-four hours later the cells were split and allowed to grow an additional $24 \mathrm{~h}$ before harvesting and immunoblot detection with an anti-FXR1P antibody (2107), followed by eIF5 antibody to control for loading. Reduction in FXR1P expression was quantified using densitometry (Image J) and, after normalization to eIF5, calculated as a percent decrease compared with control.

\section{Overexpression of miR-367 mimic}

HEK293 and HeLa cells were plated at $2 \times 10^{5}$ in 12-well dishes. Twenty-four hours after plating, cells were transfected with $50 \mu \mathrm{L}$ of $2 \mu \mathrm{M}$ double-stranded miR-367 mimic (Dharmacon) using Lipofectamine2000 transfection reagent (Invitrogen). Control cells were mock transfected using Lipofectamine2000, and each sample was compared with an untreated control or an irrelevant siRNA. After 72 or $96 \mathrm{~h}$, cells were harvested and immunoblots were probed with anti-FXR1P 2107 and then eIF5 antibody to control for loading. Reduction in FXR1P expression was quantified using densitometry (Image J) and, after normalization to eIF5, calculated as a percent decrease compared with control. Significance of reduction was determined using Student's $t$-test.

\section{ACKNOWLEDGMENTS}

We thank fellow laboratory members Claudia Winograd and Miri Kim for their advice on statistics and the luciferase assay, respectively. We also thank Dr. Ashish Lal, Dr. Leah Matzat, and members of our laboratory for reading this manuscript and for providing helpful comments. The DT40 cell line was a kind gift from Dr. T. Fukagawa of the National Institute of Genetics in Japan. We would also like to thank Dr. Andre Hoogeveen for the FXR1P 2107 antibody. This work was supported in part by the William and Enid Rosen Fellowship from the National Fragile X Foundation awarded to A.C., the National Institutes of Health, University of Illinois at Urbana-Champaign Developmental Psychobiology and Neurobiology Training Grant [\#2T32HD007333-21] to E.B., the Spastic Paralysis Research Foundation of the Illinois-Eastern Iowa District of Kiwanis International to S.C., and the UIUC Campus Research Board award to S.C.

Received December 1, 2009; accepted April 26, 2010.

\section{REFERENCES}

Abdelmohsen K, Lal A, Kim H, Gorospe M. 2007. Post-transcriptional orchestration of an anti-apoptotic program by HuR. Cell Cycle 6: $1288-1292$.

Abdelmohsen K, Srikantan S, Kuwano Y, Gorospe M. 2008. miR-519 reduces cell proliferation by lowering RNA-binding protein HuR levels. Proc Natl Acad Sci 105: 20297-20302.

Bakker C, de Diego Oter Y, Bontekoe C, Raghoe P, Luteijn T, Hoogeveen A, Oostra B, Willemsen R. 2000. Immunocytochemical and biochemical characterization of FMRP, FXR1P, and FXR2P in the mouse. Exp Cell Res 258: 162-170. 
Bartel D. 2004. MircroRNAs: Genomics, biogenesis, mechanism, and function. Cell 116: 281-297.

Bartel D. 2009. MircroRNAs: Target recognition and regulatory functions. Cell 136: 215-233.

Buerstedde J, Takeda S. 1991. Increased ratio of targeted to random integration after transfection of chicken B cell lines. Cell 67: 179-188.

Cheever A, Ceman S. 2009. Phosphorylation of FMRP inhibits association with Dicer. RNA 15: 1-5.

Chen J, Mandel E, Thomson J, Wu Q, Callis T, Hammond S, Conlon F, Wang D. 2006. The role of microRNA-1 and microRNA-133 in skeletal muscle proliferation and differentiation. Nat Genet 38: 228-233.

Chen J, Murchison E, Tang R, Callis T, Tatsuguchi M, Deng Z, Rojas M, Hammond S, Schneider M, Selzman C, et al. 2008. Targeted deletion of Dicer in the heart leads to dilated cardiomyopathy and heart failure. Proc Natl Acad Sci 105: 2111-2116.

Chen J, Callis T, Wang D. 2009. microRNAs and muscle disorders. J Cell Sci 122: 13-20.

Cherry J, Karschner V, Jones H, Pekala P. 2006. HuR, an RNAbinding protein, involved in the control of cellular differentiation. In Vivo 20: 17-23.

Chi S, Zang J, Mele A, Darnell R. 2009. Argonaute HITS-CLIP decodes microRNA-mRNA interaction maps. Nature 460: 479-486.

Coy J, Sedlacek Z, Bachner D, Hameister H, Joos S, Lichter P, Delius H, Poustka A. 1995. Highly conserved 3' UTR and expression pattern of FXR1 points to a divergent gene regulation of FXR1 and FMR1. Hum Mol Genet 4: 2209-2218.

Davidovic L, Sacconi S, Bechara E, Delplace S, Allegra M, Desnuelle C, Bardoni B. 2008. Alteration of expression of muscle specific isoforms of the fragile X related protein (FXR1P) in facioscapulohumeral muscular dystrophy patients. J Med Genet 45: 679-685.

Devys D, Lutz Y, Rouyer N, Bellocq J, Mandel J. 1993. The FMR-1 protein is cytoplasmic, most abundant in neurons and appears normal in carriers of fragile X premutation. Nat Genet 4: 335-340.

Doran J, Strauss W. 2007. Bioinformatic trends for the determination of miRNA-target interactions in mammals. DNA Cell Biol 26: $353-$ 360.

Dube M, Huot M, Khandjian E. 2000. Muscle specific fragile X related protein 1 isoforms are sequestered in the nucleus of undifferentiated myoblast. BMC Genet 1: 4. doi: 10.1186/1471-2156-1-4.

Eisenberg I, Alexander M, Kunkel L. 2009. miRNAs in normal and diseased skeletal muscle. J Cell Mol Med 13: 2-11.

Feng Y, Absher D, Eberhart DE, Brown V, Malter HE, Warren ST. 1997. FMRP associates with polyribosomes as an mRNP, and the I304N mutation of severe fragile $\mathrm{X}$ syndrome abolishes this association. Mol Cell 1: 109-118.

Filipowicz W, Bhattacharyya S, Sonenberg N. 2008. Mechanisms of post-transcriptional regulation by microRNAs: Are the answers in sight? Nature 9: 102-114.

Fukagawa T, Nogami M, Yoshikawa M, Ikeno M, Okazaki T, Takami Y, Nakayama T, Oshimura M. 2004. Dicer is essential for formation of the heterochromatin structure of vertebrate cells. Nat Cell Biol 6: 784-791.

Gorospe M. 2003. HuR in the mammalian genotoxic response: Posttranscriptional multitasking. Cell Cycle 2: 412-414.

Grimson A, Farh K, Johnston W, Garrett-Engele P, Lim L, Bartel D. 2007. MicroRNA targeting specificity in mammals: Determinants beyond seed pairing. Mol Cell 27: 91-105.

Gu S, Jin L, Zhang F, Sarnow P, Kay M. 2009. Biological basis for restriction of microRNA targets to the $3^{\prime}$ untranslated region in mammalian mRNAs. Nat Struct Mol Biol 16: 144-150.

Hendrickson D, Hogan D, Herschlag D, Ferrell J, Brown P. 2008. Systematic identification of mRNAs recruited to argonaute 2 by specific microRNAs and corresponding changes in transcript abundance. PLoS One 3: e2126. doi: 10.1371/journal.pone.0002126.

Huot M, Bisson N, Davidovic L, Mazroui R, Labelle Y, Moss T, Khandjian E. 2005. The RNA-binding protein fragile X-related 1 regulates somite formation in Xenopus laevis. Mol Biol Cell 16: $4350-4361$.
Hurteau G, Carlson J, Spivack S, Brock G. 2007. Overespression of the microRNA hsa-miR-200c leads to reduced expression of transcription factor 8 and increased expression of E-cadherin. Cancer Res 67: 7972-7976.

Hutvagner G, McLachlan J, Pasquinelli A, Balint E, Tuschl T, Zamore P. 2001. A cellular function for the RNA-interference enzyme Dicer in the maturation of the let-7 small temporal RNA. Science 293: 834-838.

Jackson A, Burchard J, Schelter J, Chau B, Cleary M, Lim L, Linsley P. 2009. Widespread siRNA 'off-target' transcript silencing mediated by seed region sequence complementarity. RNA 12: 1179-1187.

Jin P, Zarnescu D, Ceman S, Nakamoto M, Mowrey J, Jongens T, Nelson D, Moses K, Warren S. 2004. Biochemical and genetic interaction between the fragile $\mathrm{X}$ mental retardation protein and the microRNA pathway. Nat Neurosci 7: 113-117.

Khandjian E, Fortin A, Thibodeau A, Tremblay S, Cote F, Devys D, Mandel J, Rousseau F. 1995. A heterogeneous set of FMR1 proteins is widely distributed in mouse tissues and is modulated in cell culture. Hum Mol Genet 4: 783-789.

Khandjian E, Corbin F, Woerly S, Rousseau F. 1996. The fragile X mental retardation protein is associated with ribosomes. Nat Genet 12: 91-93.

Khandjian EW, Bardoni B, Corbin F, Sittler A, Giroux S, Heitz D, Tremblay S, Pinset C, Montarras D, Rousseau F, et al. 1998. Novel isoforms of the fragile $\mathrm{X}$ related protein FXR1P are expressed during myogenesis. Hum Mol Genet 7: 2121-2128.

Kirkpatrick L, McIlwain K, Nelson D. 1999. Alternative splicing in the murine and human FXR1 genes. Genomics 59: 193-202.

Kirkpatrick L, Mcllwain K, Nelson D. 2001. Comparative genomic sequence analysis of the FXR gene family: FMR1, FXR1, and FXR1. Genomics 78: 169-177.

Lal A, Navarro F, Maher C, Maliszewski L, Yan N, O’Day E, Chowdhury D, Dykxhoorn D, Tsai P, Hofmann O, et al. 2009. miR-24 inhibits cell proliferation by targeting E2F2, MYC, and other cell-cycle genes via binding to 'seedless' 3'UTR microRNA recognition elements. Mol Cell 35: 610-625.

Landgraf P, Rusu M, Sheridan R, Sewer A, Iovino N, Aravin A, Pfeffer S, Rice A, Kamphorst A, Landthaler M, et al. 2007. A mammalian microRNA expression atlas based on small RNA library sequencing. Cell 129: 1401-1414.

Lewis B, Burge C, Bartel D. 2005. Conserved seed pairing, often flanked by adenosines, indicates that thousands of human genes are microRNA targets. Cell 120: 15-20.

Lopez de Silanes I, Lal A, Gorospe M. 2005. HuR: Post-transcriptional paths to malignancy. RNA Biol 2: 11-13.

Lytle J, Yario T, Steitz J. 2007. Target mRNAs are repressed as efficiently by microRNA-binding sites in the $5^{\prime}$ UTR as in the 3' UTR. Proc Natl Acad Sci 104: 9667-9672.

Majoros W, Ohler U. 2007. Spatial preferences of microRNA targets in $3^{\prime}$ untranslated regions. BMC Genomics 8: 152. doi: 10.1186/ 1471-2164-8-152.

Mazroui R, Huot M, Tremblay S, Boilard N, Labelle Y, Khandjian E. 2003. Fragile X Mental Retardation protein determinants required for its association with polyribosomal mRNPs. Hum Mol Genet 12: 3087-3096.

Mientjes E, Willemsen R, Kirkpatrick L, Nieuwenhuizen I, HoogeveenWesterveld M, Verweij M, Reis S, Bardoni B, Hoogeveen A, Oostra B, et al. 2004. Fxr1 knockout mice show a striated muscle phenotype: implications for Fxrlp function in vivo. Hum Mol Genet 13: 1291-1302.

Nilsen T. 2007. Mechanisms of microRNA-mediated gene regulation in animal cells. Trends Genet 23: 243-249.

Padje S, Chaudhry B, Severijnen L-A, Linde H, Mientjes E, Oostra B, Willemsen R. 2009. Reduction in fragile $\mathrm{X}$ related 1 protein causes cardiomyopathy and mucular dystrophy in zebrafish. J Exp Biol 212: 2564-2570.

Price D, Zhang F, Ashley C, Warren S. 1996. The chicken FMR1 gene is highly conserved with a CCT $5^{\prime}$-untranslated repeat and encodes an RNA-binding protein. Genomics 31: 3-12. 
Rigoutsos I. 2009. New tricks for animal microRNAs: Targeting of amino acid coding regions at conserved and nonconserved sites. Cancer Res 69: 3245-3248.

Ruan H, Brown C, Morris D. 1997. Analysis of ribosome loading onto mRNA species: Implications for translational control. In $m R N A$ formulation and function (ed. J Richter), pp. 305-321. Academic, New York.

Scott G, Goga A, Bhaumik D, Berger C, Sullivan C, Benz C. 2007. Coordinate suppression of ERBB2 and ERBB3 by enforced expression of micro-RNA miR-125a or miR-125b. J Biol Chem 282: $1479-1486$.

Siomi M, Siomi H, Sauer W, Srinivasan S, Nussbaum R, Dreyfuss G. 1995. FXR1, and autosomal homolog of the fragile X mental retardation gene. EMBO J 14: 2401-2408.

Stetler A, Winograd C, Sayegh J, Cheever A, Patton E, Zhang X, Clarke S, Ceman S. 2006. Identification and characterization of the methyl arginines in the fragile $\mathrm{X}$ mental retardation protein Fmrp. Hum Mol Genet 15: 87-96.

Terracciano A, Chiurazzi P, Neri G. 2005. Fragile X syndrome. Semin Med Genet 137C: 32-37.
Vasudevan S, Steitz J. 2007. AU-rich-element-mediated upregulation of translation by FXR1 and Argonaute 2. Cell 128: 1105-1118.

Vasudevan S, Tong Y, Steitz J. 2007. Switching from repression to activation: MicroRNAs can up-regulate translation. Science 318: 1931-1934.

Wienholds E, Plasterk R. 2005. MicroRNA function in animal development. FEBS Lett 579: 5911-5922.

Winograd C, Clayton D, Ceman S. 2008. Expression of fragile X mental retardation protein within the vocal control system of developing and adult male zebra finches. Neuroscience 157: 132-142.

Xiao C, Calado D, Galler G, Thai T, Patterson H, Wang J, Rajewsky N, Bender T, Rajewsky K. 2007. MiR-150 controls B cell differentiation by targeting the transcription factor c-Myb. Cell 131: 146159.

Yekta S, Shih I, Bartel D. 2004. MicroRNA-directed cleavage of HOXB8 mRNA. Science 304: 594-596.

Zhang Y, O'Connor J, Siomi M, Srinivasan S, Dutra A, Nussbaum R, Dreyfuss G. 1995. The fragile X mental retardation syndrome protein interacts with novel homologs FXR1 and FXR2. EMBO J 14: $5358-5366$. 

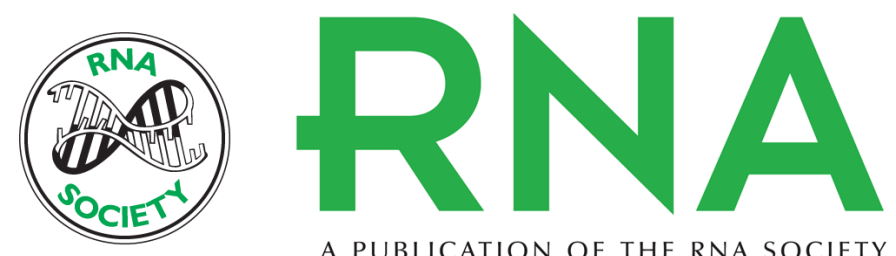

A PUBLICATION OF THE RNA SOCIETY

\section{Fragile $\mathrm{X}$ protein family member FXR1P is regulated by microRNAs}

Anne Cheever, Ernest Blackwell and Stephanie Ceman

RNA 2010 16: 1530-1539 originally published online June 2, 2010

Access the most recent version at doi:10.1261/rna.2022210

\section{References This article cites 58 articles, 15 of which can be accessed free at: http://rnajournal.cshlp.org/content/16/8/1530.full.html\#ref-list-1}

License

Email Alerting Receive free email alerts when new articles cite this article - sign up in the box at the Service top right corner of the article or click here.

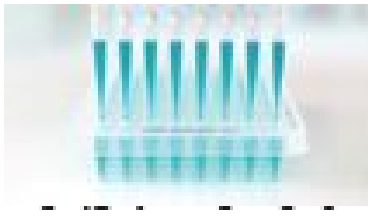

